\title{
EMF Estimation of Over Head Transmission Line Using CS Algorithm with Aid of NFC
}

\author{
P. Sivakami ${ }^{1}$ and P. Subburaj ${ }^{2}$ \\ ${ }^{1}$ Department of Electrical and Electronics Engineering, Unnamalai Institute of Technology, \\ Kovilpatti, Thoothukudi District, Tamilnadu \\ ${ }^{2}$ Department of Electrical and Electronics Engineering \\ National Engineering College, Kovilpatti \\ sivakamip0877@gmail.com
}

\begin{abstract}
In this paper, the cuckoo search (CS) algorithm - neuro fuzzy controller (NFC) technique is proposed for estimating the electric and magnetic field (EMF) of over head transmission line (OHTL). In the proposed method, the CS algorithm is used to optimize the conductor spacing (arrangements) and to minimize the EMF. From the optimized arrangement of the conductors, the EMF of the transmission lines is evaluated and formed as the datasets. Then the optimized data set values are applied to the input of the NFC. Subsequently, the coordinates are given to the input of the NFC which predicts their corresponding EMF data. The proposed method is implemented in the MATLAB working platform. For testing the performance of the proposed estimation methods, two overhead transmission line models with $110 \mathrm{kV}$ and $400 \mathrm{kV}$ respectively are considered. In order to verify the effectiveness of the proposed method this is compared with the CS algorithm-ANN controller.
\end{abstract}

Keywords: EMF, CS algorithm, NFC, transmission lines, conductor and coordinates

\section{Introduction}

The magnetic fields provoked in the vicinity of open air type substations are of great anxiety for possible health effects to people working inside these substations, or people leaving close by [1]. The only manner to be safest from the electric and magnetic fields publicity generated in open air type substations is to at least make certain that one is pictured to electric and magnetic fields inside the existing safety guideline limits [2]. Newly, environmental exposure to synthetic EMFs has been gradually increasing as the growing demand for electricity, and ever advancing technologies and varies in social behaviour have formed more and more artificial sources [3] [4]. To the improvement of transmission lines in operation range, which produce very strongly electric field in their near locality [5]. Consequently, it is significant to address the issue of environmental conditions in and around the overhead transmission line [6] [7].

Various concerns are there regarding possible biological effects of low-frequency electric and magnetic fields [11]. The calculation of the electric and magnetic field values within a substation is a hard task [8]. Owing to the difficulty arrangements of geometry of the bus bars, power transformers, insulators, voltage and current measurement transformers and increase of connected power lines within the covered space [9] [11]. As a result, it is needed to apply a dimensional based coordinate approach to the computation and study of the electromagnetic field [10]. Artificial intelligence techniques have been effectively used to an estimate the overhead transmission line electromagnetic field during latest years [14]. Fuzzy logic is applied for magnetic field estimations at transformer substations [12]. In fuzzy logic, the overhead line condition index, which symbolizes the numerical expression of the degree of its condition aggravation and the basis for the choice of the maintenance actions to be taken [15].

For the adjustment of the fuzzy parameters the genetic algorithm has been proposed [16] [17]. Artificial neural networks are addressed in order to give precise solutions to high voltage transmission line problems [18]. ANFIS and RBF network have been effectively employed to a

Received: October $12^{\text {th }}, 2015$. Accepted: September $23^{\text {rd }}, 2016$ 
number of engineering problems during latest years [19]. The architecture and learning procedure of fuzzy interference system executed in the framework of adaptive networks. One of ANFIS functions is modelling difficult nonlinear functions by a set of fuzzy rules. Neural network and fuzzy logic system are common approximations. The RBF neural network as well has the ability of common approximation [20]. These artificial intelligence techniques are effectively used for electromagnetic fields estimation around an overhead power transmission line [13]. In this paper, CS algorithm with aid of NFC is proposed to estimate the EMF of transmission line and also find the optimal arrangement of conductors. The detailed description of the proposed technique is presented in Section 3. Prior to that, the recent research works are presented in Section 2. The experimental results and discussion are given in Section 4. Finally, the Section 5 concludes the paper.

\section{Recent Research Works: A Brief Review}

In literature different research works are already presented which based on electric and magnetic field estimation in overhead transmission line by AI techniques, noncontact technology and etc. In this segment, a generic reassess performed.

Jasna Radulovic et al. [21] have distressed the chance that the revelation to very low frequency electromagnetic fields from power lines may have dangerous effects on human and living organisms. They have been offered approach based on the employ of both feed forward neural network (FNN) and adaptive network based fuzzy inference system (ANFIS) to calculate approximately electric and magnetic fields around an overhead power transmission lines. Using the results obtained from the earlier research an FNN and ANFIS is employed to reproduce this problem that was coached. It was demonstrated that suggested approach makes certain satisfactory precision and could be a competent tool and constructive substitute for such examinations.

A calculation of the 3-D magnetic and electric fields in terms of the influence of a steel tower has been offered by Zemljaric. B et al. [22]. The method joins the magnetic and electric quasi-static field calculation approach through the suitable electric circuit model and equations. By means of this approach the electric field potentials of the tower elements are not equal to zero and the involvement of the induced electric field was taken into report. The potentials mainly rely on the value of the current running through the tower elements from the top to the ground. The electric field values for dissimilar climbing routes on the tower were computed and compared with reference values in order to forecast an optimal climbing route on the tower.

Jasna Radulovic et al. [23] have explained in detail the design of radial basis neural network models in order to assess magnetic field of power transmission lines. The network was coached by gradient descent algorithm. The results attained according to the suggested NRBF method were very near to those computed by means of Charge simulation method, which obviously implies that the suggested method makes certain acceptable precision and pleasing convergence. The benefit of the employ of NRBF in relation to conventional methods for computation of magnetic fields of power lines is that neural network was necessary to be coached only once. NRBF could be employed to find out the magnetic field distribution after the achievement of training in a novel geometry varying from the geometries applied for training. Maximal absolute fault is less than $4 \%$ when NRBF was employed. These developed NRBF model makes it feasible to find out the magnetic fields easier, while offering significant diminution of analysis time.

A noncontact technology of operation state monitoring has been suggested by Xu Sun et al. [24] based on magnetic field sensing for high voltage transmission lines, which could at the same time calculate both electrical and spatial parameters in real time. This technology was obtained from research on the magnetic field distribution at the ground level when the transmission lines function in different states, together with sagging, galloping, and current imbalance. Two distinctive models of high-voltage three phase transmission lines were 
reproduced, and the resulting magnetic fields were worked out. The association among the magnetic field variations and the operation states were examined. Based on such correlation, a source reconstruction method was proposed to renovate the spatial and electrical parameters from the magnetic field derived by the overhead transmission lines. The reconstruction effects for the $500-\mathrm{kV}$ and $220-\mathrm{kV}$ transmission lines validate the possibility and practicality of this noncontact transmission line monitoring technology based on magnetic field sensing.

F. Munoz et al. [25] have conversed the application of particular Artificial Intelligence techniques offers a competent solution to the problem of characterizing the magnetic field of a high voltage overhead transmission line, and is a substitute to the expensive procedure of direct measurements, which needs equipment and time, to the employ of complex numerical methods of a very precise scope, or of simply gaining a theoretical value calculated by means of analytical procedures which give up the quality of the solution in support of making simpler the computations. They offered an execution which based on a hybrid algorithm in which the best solutions presented by a metaheuristics define the initial simplex for the application of the Nelder-Mead Method, which as a local search method allows a calculation-intensive search. In order to authenticate the quality of the results produced by this hybrid implementation, the estimates attained were compared with measured values and with values attained using analytical procedures.

The reassess of the current research works demonstrates that, the extra high voltage transmission line produces very low frequency electric and magnetic fields. Different human health hazards are related with this low frequency fields as the low frequency field's prediction participates an important role. Many methods have been suggested for categorizing the frequency of the signal such as Maxwell's equation, analytical method, empirical method and etc. For calculating the magnetic field Maxwell's equation permits only very easy geometries to direct integration. The benefits of analytical method regarding other techniques which presented information on the subject of the parameters so as to have an outcome on the magnetic field produced by overhead lines and tolerate examining original configurations in a simpler approach. In investigative methods, the computed magnetic field value is just appropriate at large distances from the line assessed to the distances among conductors. As a result, this method is appropriate only for electrical lines with multilateral regularity, apart from for in the smallest amount configuration. The process of Empirical method is based on the features measurement of high voltage transmission line under electromagnetic environment. However, the effect of this procedure is high-priced for both time and equipment. Hence by the difference of electrical and environmental conditions the measurement is affected. To conquer problem related with characterization over head line signal in the magnetic environment, the artificial intelligence technique is applied. In the paper, an optimization algorithm based artificial intelligence technique is applied for estimating the EMF generated by overhead transmission line. The detailed explanation is described in the following section.

\section{EMF Calculation of Over Head Transmission Line}

The magnetic field around a three-phase line can be computed by superimposing the individual contribution of the current of each phase conductor and taking into consideration the return currents through the earth. The magnetic field intensity at the point ' $\mathrm{j}$ ' is achieved by taking into account the contribution of all ' $\mathrm{N}$ ' conductors, presuming parallel lines over a flat earth [26]. A line conductor is located at $\left(x_{i}, y_{i}\right)$ with electric current of Ii. The geometry is taken into consideration to estimate the magnetic field at $\left(x_{j}, y_{j}\right)$ because of the fact that the phase conductor takes the complicated images. The magnetic field is estimated by means of the formula as per Equation 1 . 


$$
H_{e}^{j}=\sum_{i}^{N}\left(\frac{I_{i}}{r_{i j} 6.28}\right) u_{i j}+\sum_{i}^{N}\left(\frac{-I_{i}}{r_{i j}^{\prime} 6.28}\right)\left(\left(1+\frac{16}{3\left(r^{\prime}\right)^{4}}\right)\right) u_{i j}^{\prime}
$$

Note that $r_{i j}$ represents the distance between line conductor and field point, while $r_{i j}$ indicates the distance between the complex image of line conductor, through earth, and the field point.

$$
\begin{aligned}
& \gamma=\sqrt{j \omega \mu(\sigma+j \omega \varepsilon)} \\
& u_{i j}=\left(\frac{y_{i}-y}{r_{i j}}\right) u_{x}-\left(\frac{x_{i}-x}{r_{i j}}\right) u_{y} \\
& u_{i j}^{\prime}=\left(\frac{y_{j}+y_{i}+\left(\frac{2}{\gamma}\right)}{r_{i j}^{\prime}}\right) u_{x}-\left(\frac{x_{i}-x_{j}}{r_{i j}^{\prime}}\right) u_{y}
\end{aligned}
$$

In the above equation, $\sigma, \varepsilon, \mu$ represent the conductivity, permittivity, and permeability of the earth. The phase current, and unit vectors are symbolized as $I_{i}, u_{x}, u_{y}$. By means of the above-mentioned equation, the magnetic field is computed. For the purpose of evaluation of the electric field, the following equation is employed.

$$
\begin{aligned}
& E_{e}^{j}=\left(\frac{C V}{4 \pi \varepsilon_{0}}\right)\left[\frac{2(y-h) u_{y}+2\left(x-x_{0}\right) u_{x}}{(y-h)^{2}+\left(x-x_{0}\right)^{2}}-\frac{2(y+h) u_{y}+2\left(x-x_{0}\right) u_{x}}{(y+h)^{2}+\left(x-x_{0}\right)^{2}}\right] \\
& C=\frac{1}{18 \ln (G M D / r)} \\
& G M D=\left(D_{A B} D_{B C} D_{A C}\right)^{\frac{1}{3}}
\end{aligned}
$$

Where $r$ denotes the conductor radius and GMD represents the geometric mean distance. Thereafter, the electric field of the communication line is estimated.

\section{A. EMF Estimation of transmission line using proposed method}

In this paper, an innovative method is used to estimate the EMF of the OHTL systems. The proposed method is the CS algorithm with aid of NFC. Here, the CS algorithm is used to optimize the conductor spacing and minimize the EMF. Initially, the $\mathrm{x}$ and $\mathrm{y}$ co-ordinates, conductor size, voltage, current etc. are given as the inputs of the proposed method. Then the EMF is evaluated according to their optimal conductor arrangements. Subsequently, the optimized datasets are formed by using the CS algorithm and are given as the input to the NFC. 
The NFC controller is used to predict the corresponding EMF data for the given input. The detailed explanation of the proposed CS algorithm combined with the NFC technique is described in the following section 3.1. The block diagram of the proposed technique is illustrated in Figure 1.

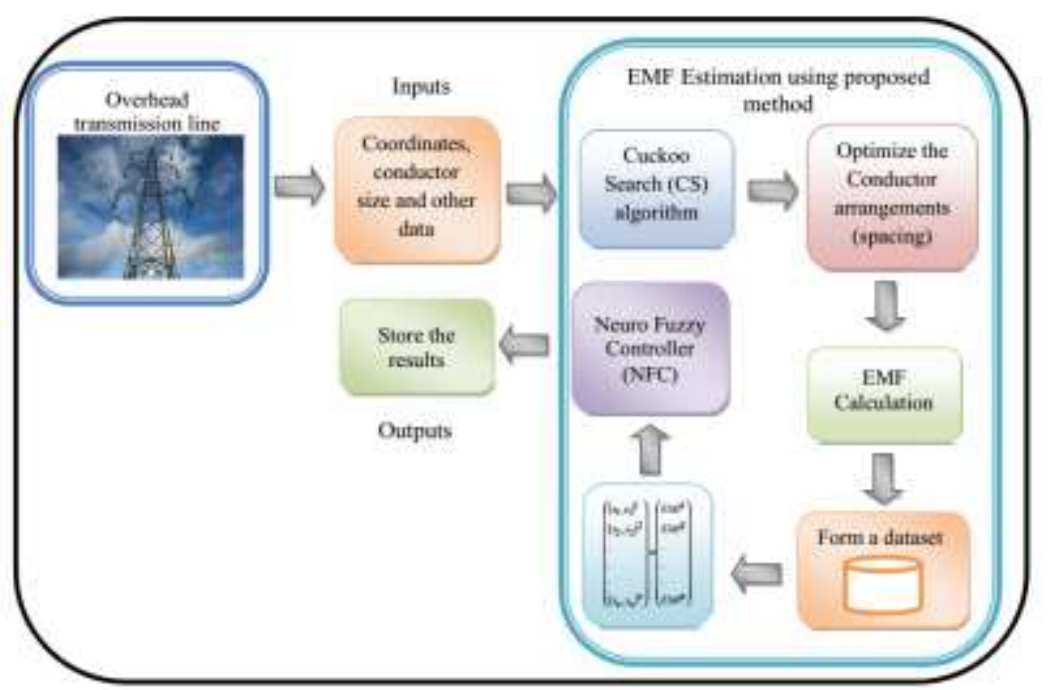

Figure 1. EMF estimation of OHTL using proposed method

\section{A.1. Optimal conductor arrangements and EMF dataset generation using CS algorithm}

Cuckoo search technique, in essence, is a meta-heuristic method enthused by the breeding trend of the cuckoos and is easy to implement. In the cuckoo search, there is a host of nests. Each and every egg accounts for a solution and the egg of a Cuckoo specifies a new solution. The new and better solution displaces the worst solution in the nest [27]. Now, the cuckoo search technique is employed for reducing the EMF of the transmission line system to optimize the conductor arrangements. The inputs of CS algorithm are represented by the $\mathrm{x}$ and $\mathrm{y}$ coordinates, voltage, current, conductor size etc. The fitness function is estimated for ach iteration. The EMF data represents the output of the innovative technique, An extensive account of the novel method is elegantly explained hereunder.

Step 1: Define the Objective function

In this section, the objective function is considered as an optimization problem. Here, the optimization problem is defined as follows:

$$
O_{f}=\min \left(E M_{F}\right)
$$

Where, $\min \left(E M_{F}\right)=\min \left(H_{e}^{j}, E_{e}^{j}\right)$. The objective function $\left(O_{f}\right)$ has to be minimized by arranging the conductor in an optimal way in the search space of its limits.

\section{Step 2: Describe the Initialization Process}

In this section, the host nests are randomly initiated. Here, the nest is an array of size ' $n$ '.

$$
\begin{aligned}
& N_{k}=\left\{n_{1}, n_{2}, \ldots \ldots . . n_{n}\right\} \\
& C_{k}=\left\{c_{1}, c_{2}, \ldots \ldots . . c_{n}\right\}
\end{aligned}
$$


Here, $N_{k}$ and $C_{k}$ are denoted as the $\mathrm{x}$ and y coordinates respectively. Then each nest $n_{n}$ is a solution vector to the optimization problem. It can hold $n$ number of variables, which are optimized so as to minimize the objective function.

Step 3: Establish the new cuckoo generation

A cuckoo randomly generates new solutions by using the Levy function and determines the quality of solutions. The Levy function $(\operatorname{Levy}(\lambda))$ is represented as follows:

$$
N_{K}^{t+1}=N_{k}^{t}+\alpha \oplus \operatorname{Levy}(\lambda)
$$

The Cuckoo is evaluated using the objective function to determine the quality of the solutions.

Step 4: Compute the Fitness for all inputs

Here, the fitness function ( $F F$ ) of all the inputs are evaluated by using the following formula,

$$
(F F)=\min \left(E M_{F}\right)
$$

Where, the electric and magnetic field depend on the conductor arrangements and the $\mathrm{x} \& \mathrm{y}$ coordinates. If the fitness function is minimized then the current solution is saved as an optimal solution. Otherwise, the previous solution is kept as the best solution.

\section{Step 5: Discard the worst nest}

In this part, the worst nests are discarded based on their probability $p_{a}$ values and the new ones are built using the equation (1). Subsequently, the best solutions are ranked based on their quality. Then the present best solutions are identified as the optimal solutions.

\section{Step 6: Terminate the process}

This process is repeated until the termination iteration is reached. The output is categorized according to their inputs and the corresponding output is noted. The output of the Cuckoo search algorithm is furnished as follows:

$$
\left(\begin{array}{l}
\left(x_{1}, y_{1}\right)^{1} \\
\left(x_{2}, y_{2}\right)^{2} \\
\cdot \\
\cdot \\
\cdot \\
\left(x_{n}, y_{n}\right)^{n}
\end{array}\right)=\left(\begin{array}{l}
E M F^{1} \\
E M F^{2} \\
\cdot \\
\cdot \\
E M F^{n}
\end{array}\right)
$$

From the output of the CS algorithm, the conductor arrangement is optimized and the optimal set of the minimum EMF of the transmission line system is evaluated. The Flow chart of the CS algorithm is illustrated in Figure 2. 


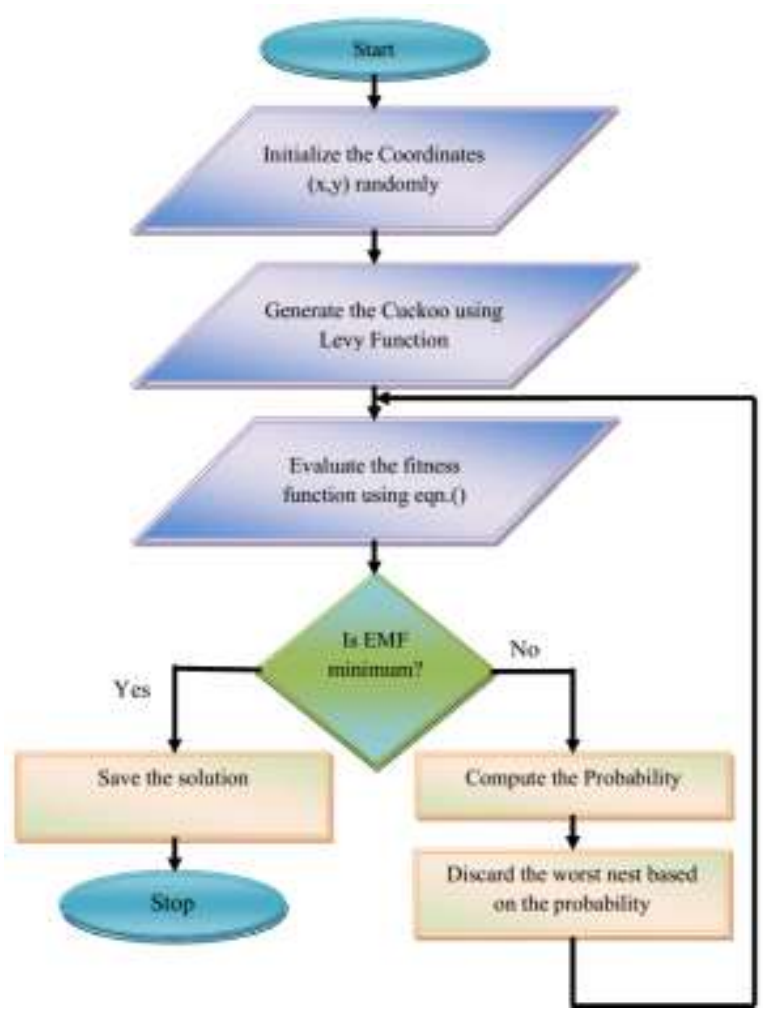

Figure 2. Flow chart of proposed CS algorithm

\section{A.2. Neuro fuzzy controller (NFC)}

Neuro fuzzy is associated with the domain of the artificial intelligence, which represents the integration of the artificial neural networks and the fuzzy logic [28]. The neural network is employed to build up the training dataset and testing for the input data applied. The innovative feed forward type neural network is employed in this regard. Normally, the neural network is home to three specific layers such as the (i) Input layer, (ii) Hidden layer and the (iii) Output layer. In the document, the optimized datasets are furnished as input to the NFC. It possesses two input layers such as the $\mathrm{x}$ and $\mathrm{y}$ coordinates a number of hidden layers and a single output i.e., the optimized electric and magnetic field (EMF) data. The function of NFC is concisely offered in the ensuing Section 3.1.2.

\section{- Neural network}

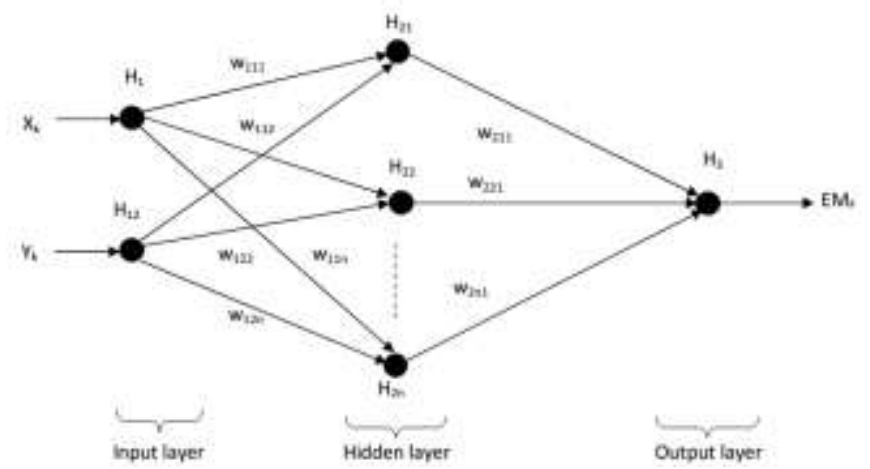

Figure 3. Structure of Neural network 
Here, two inputs are furnished as the input to the network and a single output is offered. The neural network procedure is carried out in the hidden layer. The structure of the neural network is exhibited in Figure 3.

In Figure 3, the hidden layer is given as $H_{21}, H_{22}, \ldots H_{2 N}$ and the neuron weight is labelled as $W$. The input layer to hidden neuron weight is represented by $W_{1}$ and the hidden layer to output layer weight is $W_{2}$. The proposed neural network training and weight adjustment are carried out by means of the Back Propagation (BP) process. The detailed working procedure of neural network is explained below:

Step 1: Initialize all the datasets such as the input, output and weight of the neuron. Here the inputs of the network are represented $\mathrm{x}$ and y coordinates. The optimized EMF signifies the output of the network.

Step 2: Evaluate the BP error of the input dataset.

$$
E_{1}=E M F_{T}^{N}-E M F^{N} \text { out }
$$

Where, $E M F^{N}$ represents the target output EMF, $E M F^{N}$ out the real output EMF.

Step 3: Calculate the network output using the following relation.

$$
E M F^{N} \text { out }=\sum_{n=1}^{N} W_{2 n 1} E M F^{N}(n)
$$

Where,

$$
\operatorname{EMF}^{N}(n)=\left[1+e^{\left(-W_{1 n} X(n)-W_{2 n} Y\right)}\right]^{-1}
$$

Both the equations given above represent the output layer and hidden layer activation function respectively.

Step 4: Find the new weights of all the neurons.

$W_{\text {new }}=W_{\text {old }}+\Delta W$

Where, $\Delta W$ represents the alteration in weight, which may be calculated by the following relation, $\Delta W=\gamma V_{c v}{ }^{N} E_{1} \quad \gamma$ represents the learning rate which is in the range of 0.2 to 0.5 .

Step 5: The procedure will be repeated from step 2, till the $E_{1}$ gets reduced to the minimum value.

$$
10 E_{1}<1
$$

After the process is finished, the network is well trained and generates the optimal EMF $\left(E M F^{N}\right)$. After the training procedure comes to an end, the fuzzy rules are created, which comprises three specific phases such as the fuzzification, decision making and defuzzification, which are employed to generate the optimal EMF $\left(E M F^{N}\right)$. The fuzzy rules creation is detailed in the ensuing Section 3.1.2.2. 


\section{- Development of training dataset using fuzzy rules}

The functioning of the controller is invariably dependent on the fuzzy rules. Now, the fuzzy rules are created by employing the Genfis2. The fuzzy controller comprises three phases which are detailed below:
a. Fuzzification
b. Decision making
c. Defuzzification

In the course of the fuzzification procedure, the crisp values are transformed into fuzzy value. The output is forwarded to the decision making block, which comprise of a group of regulations. By effectively employing the fuzzy rules the decision for the suitable output is produced, which is furnished to the defuzzification procedure. Defuzzification represents the inverse procedure of the fuzzification.

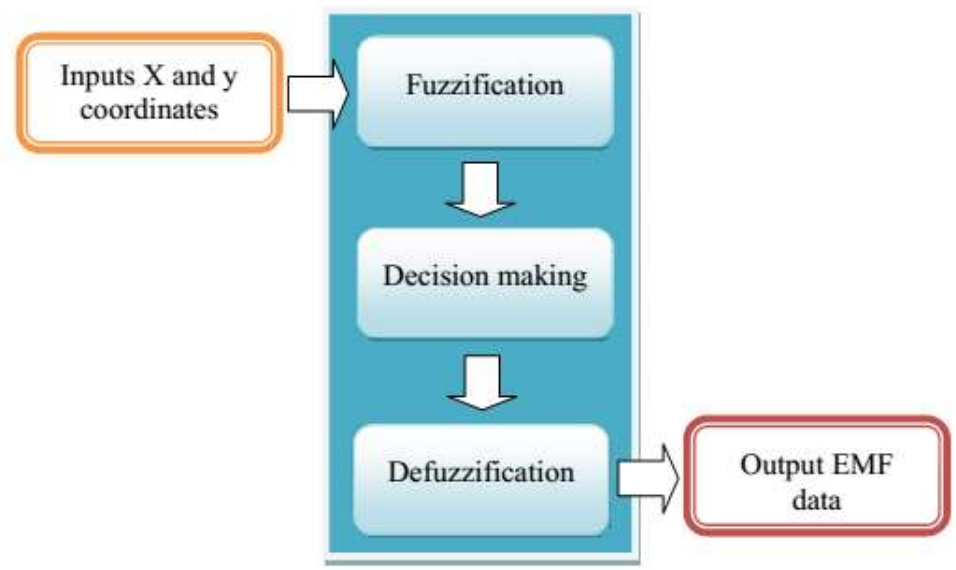

Figure 4. Fuzzy logic controller

These are in the rule base, which rules are tabulated in the following Table.1.

Table 1. Fuzzy Rules

\begin{tabular}{|c|c|c|c|c|c|}
\hline y & NB & NS & ZE & PS & PB \\
\hline NB & ZE & NS & NB & PB & PB \\
\hline NS & ZE & NS & NB & PB & PB \\
\hline ZE & NS & NB & ZE & PS & PB \\
\hline PS & NB & PB & NS & PS & PS \\
\hline PB & NB & PB & NS & PS & PS \\
\hline
\end{tabular}


The Table shown above illustrates the fuzzy rules, which are capable of developing the EMF. The proposed system is discussed with the NFC, by performing on the MATAB platform. The outcomes of the proposed system together with details are furnished in the ensuing Section 4.

\section{Results and discussion}

The proposed method is implemented in MATLAB working platform. Here, the CS algorithm and the NFC are used for estimating the electric \& magnetic field of the transmission line. The $\mathrm{x}$ and $\mathrm{y}$ coordinates are given to the input of CS algorithm and the EMF is estimated. Subsequently, the optimized datasets are given as input to the NF controller. In order to verify the effectiveness and robustness of the proposed method, it is compared with the CS algorithm-NN techniques. Here, the $400 \mathrm{kV}$ and $110 \mathrm{kV}$ transmission lines data [21] [23] are used to estimate the EMF. The implementation parameters of the proposed method are given in Table 2 .

Table 2. Implementation parameters

\begin{tabular}{|c|c|}
\hline Description & Values \\
\hline Conductor radius & $15 \mathrm{~mm}$ \\
\hline Current & $50 \mathrm{~A}$ \\
\hline permeability & 1 \\
\hline conductivity & 0.01 \\
\hline permittivity & 3 \\
\hline
\end{tabular}

\section{A. Performance analysis of $400 \mathrm{kV}$ OHTL}

Here, the magnetic field values for several new geometries are estimated and formed as a data set for training the neural network. Then the electric field of the transmission line values are evaluated for several new geometries. By applying the proposed method, the magnetic field dataset of $400 \mathrm{kV}$ transmission line is estimated and it is applied for training the NN which is tabulated in Table 4. And also, the electric field of $400 \mathrm{kV}$ transmission line data set is examined and tabulated in Table 5. Prior to that, the experimental results are evaluated by using the formula and are tabulated in Table 3. Similarly, the magnetic and electric field dataset for $110 \mathrm{kV}$ transmission line is determined using the proposed method and tabulated in Tables 6 and 7. Similarly, the electric and magnetic field values are determined by using the CS algorithm- NN controller.

Table 3. Training data sets for magnetic and electric field

\begin{tabular}{|c|c|c|c|c|c|c|}
\hline $\mathrm{x}$ & \multicolumn{3}{|c}{ Magnetic field } & \multicolumn{3}{c|}{ Electric field } \\
\hline $\mathrm{y}$ & 0 & 2 & 5 & 0 & 2 & 5 \\
\hline 0 & 0.176051 & 0.877975 & 0.790776 & 0.975875 & 0.945632 & 0.894799 \\
\hline 1 & 0.079679 & 0.996605 & 0.986747 & 0.988294 & 0.595727 & 0.410902 \\
\hline 2 & 0.155611 & 0.643388 & 0.268714 & 0.494047 & 0.907412 & 0.823737 \\
\hline 3 & 0.982771 & 0.027791 & 0.323179 & 0.306694 & 0.81117 & 0.41857 \\
\hline 4 & 0.063984 & 0.282935 & 0.409614 & 0.996551 & 0.960034 & 0.747199 \\
\hline 5 & 0.941139 & 0.494852 & 0.950255 & 0.076406 & 0.996258 & 0.985401 \\
\hline 6 & 0.290498 & 0.997869 & 0.106145 & 0.903384 & 0.562984 & 0.552139 \\
\hline
\end{tabular}




\begin{tabular}{|c|c|c|c|c|c|c|}
\hline 7 & 0.525132 & 0.919423 & 0.080968 & 0.92107 & 0.917502 & 0.845556 \\
\hline 8 & 0.682114 & 0.575742 & 0.227229 & 0.807989 & 0.872616 & 0.497713 \\
\hline 9 & 0.994606 & 0.108595 & 0.904407 & 0.987458 & 0.135439 & 0.775396 \\
\hline 10 & 0.66697 & 0.997503 & 0.981246 & 0.792188 & 0.914662 & 0.826793 \\
\hline 11 & 0.990318 & 0.149705 & 0.193985 & 0.949355 & 0.477385 & 0.309858 \\
\hline 12 & 0.787536 & 0.374738 & 0.605206 & 0.111116 & 0.557374 & 0.96387 \\
\hline 13 & 0.757792 & 0.979148 & 0.149491 & 0.997436 & 0.486735 & 0.619204 \\
\hline 14 & 0.894403 & 0.440025 & 0.531612 & 0.654395 & 0.86632 & 0.901596 \\
\hline 15 & 0.149373 & 0.652855 & 0.745344 & 0.992848 & 0.761951 & 0.682737 \\
\hline 16 & 0.977421 & 0.24295 & 0.99905 & 0.952554 & 0.917323 & 0.699777 \\
\hline 17 & 0.924271 & 0.757036 & 0.069857 & 0.826193 & 0.872025 & 0.99552 \\
\hline 18 & 0.99796 & 0.786246 & 0.705224 & 0.637367 & 0.740822 & 0.784396 \\
\hline 19 & 0.774564 & 0.03401 & 0.483698 & 0.738361 & 0.927111 & 0.905192 \\
\hline 20 & 0.9956 & 0.612996 & 0.254182 & 0.928919 & 0.315062 & 0.842084 \\
\hline 21 & 0.925713 & 0.729098 & 0.987965 & 0.45132 & 0.930967 & 0.791761 \\
\hline 22 & 0.93888 & 0.392145 & 0.226845 & 0.327452 & 0.918183 & 0.642574 \\
\hline 23 & 0.973896 & 0.687925 & 0.81236 & 0.380416 & 0.753364 & 0.922696 \\
\hline 24 & 0.318727 & 0.350766 & 0.35802 & 0.345369 & 0.405938 & 0.512698 \\
\hline 25 & 0.838107 & 0.859851 & 0.939482 & 0.931357 & 0.881294 & 0.274842 \\
\hline 26 & 0.773461 & 0.691476 & 0.662089 & 0.204938 & 0.982926 & 0.786779 \\
\hline 27 & 0.971154 & 0.347338 & 0.560459 & 0.787863 & 0.865527 & 0.476231 \\
\hline 28 & 0.57176 & 0.038161 & 0.573791 & 0.999679 & 0.966412 & 0.494106 \\
\hline 29 & 0.791531 & 0.160156 & 0.783029 & 0.969707 & 0.63156 & 0.996559 \\
\hline 30 & 0.290132 & 0.656576 & 0.779058 & 0.326591 & 0.433398 & 0.998374 \\
\hline 31 & 0.75535 & 0.992678 & 0.860443 & 0.879794 & 0.973652 & 0.94581 \\
\hline 32 & 0.884595 & 0.243494 & 0.529639 & 0.626537 & 0.674123 & 0.915845 \\
\hline 33 & 0.281584 & 0.769707 & 0.560112 & 0.468655 & 0.900272 & 0.345528 \\
\hline 34 & 0.960061 & 0.430719 & 0.697965 & 0.909305 & 0.999511 & 0.989999 \\
\hline 35 & 0.991292 & 0.973248 & 0.863319 & 0.989599 & 0.42909 & 0.976614 \\
\hline 36 & 0.095918 & 0.861121 & 0.994715 & 0.97512 & 0.746695 & 0.98764 \\
\hline 37 & 0.980517 & 0.974404 & 0.748794 & 0.991926 & 0.642458 & 0.990431 \\
\hline 38 & 0.41029 & 0.165633 & 0.545766 & 0.96091 & 0.910554 & 0.8872 \\
\hline 39 & 0.982462 & 0.777069 & 0.431248 & 0.93828 & 0.640141 & 0.190038 \\
\hline 40 & 0.988562 & 0.233165 & 0.542845 & 0.996382 & 0.584642 & 0.999036 \\
\hline 41 & 0.166611 & 0.942065 & 0.733387 & 0.992331 & 0.699243 & 0.911028 \\
\hline 42 & 0.775952 & 0.259098 & 0.157517 & 0.971612 & 0.995509 & 0.965895 \\
\hline 43 & 0.079015 & 0.542845 & 0.996826 & 0.787967 & 0.960885 & 0.993103 \\
\hline 44 & 0.560378 & 0.571564 & 0.707404 & 0.922549 & 0.454656 & 0.427784 \\
\hline 45 & 0.542845 & 0.735723 & 0.999274 & 0.816712 & 0.455433 & 0.392755 \\
\hline 46 & 0.968192 & 0.887957 & 0.049425 & 0.692679 & 0.361059 & 0.739193 \\
\hline 47 & 0.47736 & 0.506866 & 0.928585 & 0.970138 & 0.518676 & 0.842479 \\
\hline 48 & 0.24193 & 0.978044 & 0.364647 & 0.83949 & 0.891734 & 0.261852 \\
\hline
\end{tabular}




\begin{tabular}{|c|c|c|c|c|c|c|}
\hline 49 & 0.36348 & 0.951917 & 0.473625 & 0.337767 & 0.379851 & 0.99947 \\
\hline 50 & 0.490466 & 0.781581 & 0.227775 & 0.956565 & 0.563254 & 0.980543 \\
\hline
\end{tabular}

Table 4. Testing data sets for magnetic field (400kV OHTL)

\begin{tabular}{|c|c|c|c|c|}
\hline $\mathrm{x}$ & $\mathrm{y}$ & $\mathrm{He}$ & $\mathrm{He}(\mathrm{CS}-\mathrm{NN})$ & $\begin{array}{c}\text { He } \\
\text { (proposed method) }\end{array}$ \\
\hline 0 & 5 & 0.790776 & 0.30324 & 0.749885 \\
\hline 2 & 2 & 0.643388 & 0.730791 & 0.689639 \\
\hline 5 & 2 & 0.49484852 & 0.692206 & 0.561518 \\
\hline 13 & 0 & 0.757792 & 0.713019 & 0.722503 \\
\hline 14 & 2 & 0.440025 & 0.552434 & 0.607727 \\
\hline 16 & 0 & 0.977421 & 0.746597 & 0.730118 \\
\hline 17 & 5 & 0.069857 & 0.642169 & 0.601908 \\
\hline 20 & 0 & 0.9956 & 0.761714 & 0.927319 \\
\hline 22 & 0 & 0.93888 & 0.760479 & 0.864049 \\
\hline 23 & 0 & 0.973896 & 0.758175 & 0.821831 \\
\hline 27 & 2 & 0.341338 & 0.719527 & 0.471032 \\
\hline 33 & 0 & 0.281584 & 0.729675 & 0.813204 \\
\hline 40 & 2 & 0.233165 & 0.732004 & 0.540464 \\
\hline 46 & 5 & 0.887957 & 0.788332 & 0.535828 \\
\hline 49 & 0 & 0.36348 & 0.558246 & 0.465347 \\
\hline 50 & 2 & 0.781581 & 0.853539 & 0.818384 \\
\hline
\end{tabular}

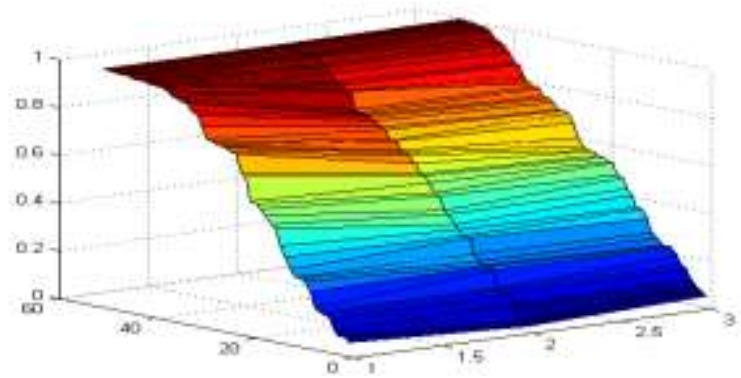

(a)

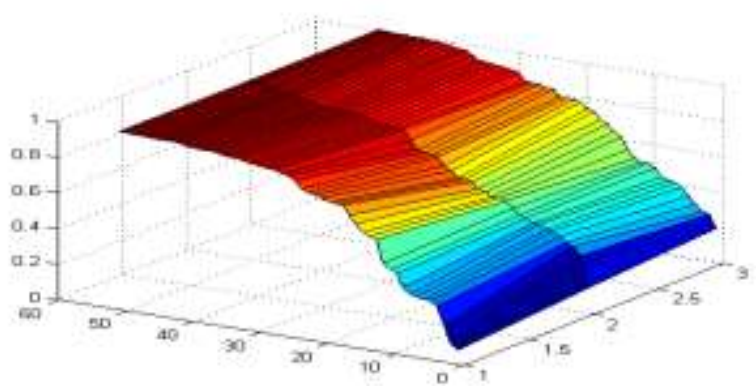

(b)

Figure 5. Analysis of (a) Magnetic field and (b) Electric field distribution in surrounding of 400 $\mathrm{kV}$ power transmission line

Tables 2 and 3 represent the magnetic field data for the training and testing of the neural network. Here, the $\mathrm{x}$ and $\mathrm{y}$ coordinates and their corresponding EMF data are $80 \%$ trained and $20 \%$ tested. Then the performance of the proposed method is determined and compared with 
the existing method. The performances of magnetic and electric fields are illustrated in the Figure 5 .

Similarly, the absolute and relative error of the electric field is calculated. The performance of the absolute and relative error values are evaluated by using the proposed and existing methods, which are illustrated in Figures 6 and 7.

Table 5. Testing data sets for electric field (400kV OHTL)

\begin{tabular}{|c|c|c|c|c|}
\hline $\mathrm{x}$ & $\mathrm{y}$ & $\mathrm{Ee}$ & $\mathrm{Ee}(\mathrm{CS}-\mathrm{NN})$ & $\begin{array}{c}\text { Ee } \\
\text { (proposed method) }\end{array}$ \\
\hline 0 & 5 & 0.894799 & 0.737746 & 0.786864 \\
\hline 2 & 2 & 0.907412 & 0.917799 & 0.738793 \\
\hline 5 & 2 & 0.996258 & 0.84324 & 0.77354 \\
\hline 13 & 0 & 0.997436 & 0.788316 & 0.720248 \\
\hline 14 & 2 & 0.86632 & 0.630977 & 0.705231 \\
\hline 16 & 0 & 0.952554 & 0.745242 & 0.694367 \\
\hline 17 & 5 & 0.99552 & 0.808164 & 0.767581 \\
\hline 20 & 0 & 0.928919 & 0.692387 & 0.618246 \\
\hline 22 & 0 & 0.327452 & 0.699699 & 0.538451 \\
\hline 23 & 0 & 0.380416 & 0.716428 & 0.528423 \\
\hline 27 & 2 & 0.865527 & 0.598921 & 0.70372 \\
\hline 33 & 0 & 0.468655 & 0.802203 & 0.739323 \\
\hline 40 & 2 & 0.584642 & 0.638523 & 0.76525 \\
\hline 46 & 5 & 0.361059 & 0.589273 & 0.53724 \\
\hline 49 & 0 & 0.337767 & 0.950802 & 0.853199 \\
\hline 50 & 2 & 0.563254 & 0.625189 & 0.507234 \\
\hline
\end{tabular}

Also, the absolute and relative error performance of the proposed method is evaluated. Similarly, the corresponding error values are calculated by using the existing method. The absolute value of the absolute error has been computed as per the following equation:

$$
\begin{aligned}
& \text { Absolute error }=\left|H_{e}-H_{e}^{C S-N F C}\right| \\
& \text { Relative error }=\left|\frac{H_{e}-H_{e}^{C S-N F C}}{H_{e}}\right|
\end{aligned}
$$




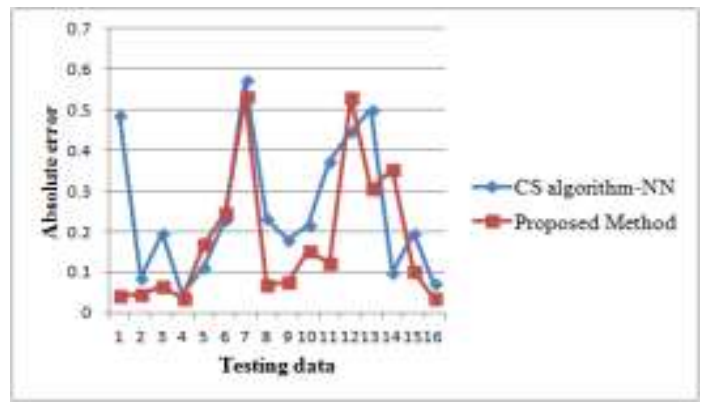

(a)

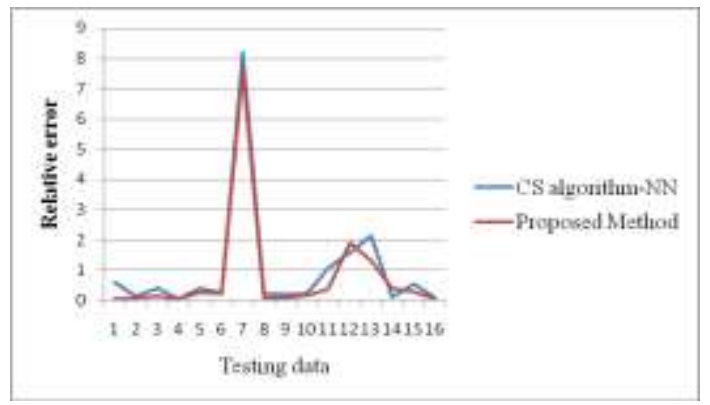

(b)

Figure 6. Comparison analysis of (a) absolute error and (b) relative error

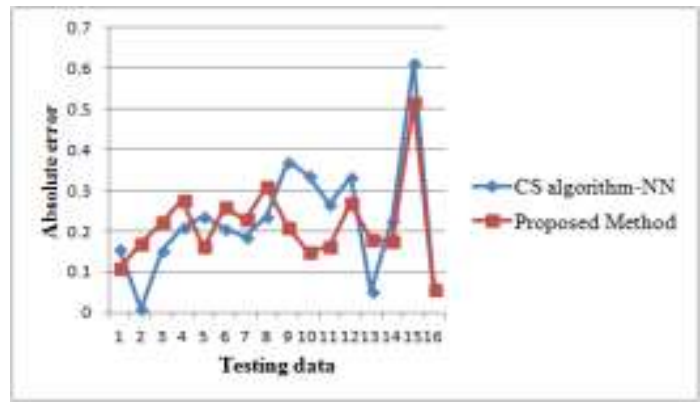

(a)

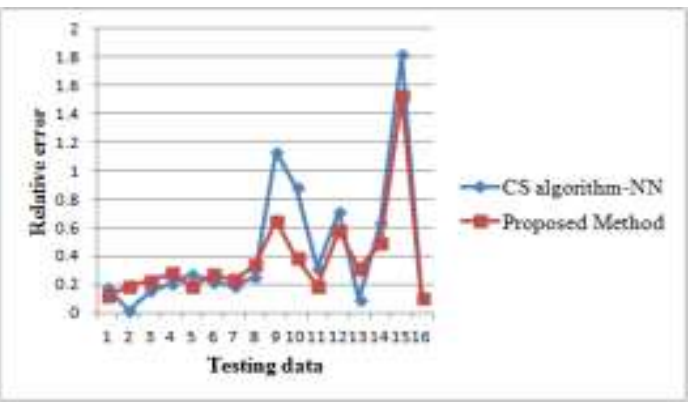

(b)

Figure 7. Comparison analysis of (a) absolute error and (b) relative error 
B. Performance analysis of $110 \mathrm{kV}$ OHTL

Table 6. Training data sets for magnetic field

\begin{tabular}{|c|c|c|c|c|c|c|}
\hline $\mathrm{x}$ & \multicolumn{3}{|c|}{ Magnetic field } & \multicolumn{3}{|c|}{ Electric field } \\
\hline $\mathrm{y}$ & 0 & 2 & 5 & 0 & 2 & 5 \\
\hline 0 & 0.156659 & 0.540718 & 0.680792 & 0.968401 & 0.765946 & 0.896318 \\
\hline 1 & 0.075306 & 0.910585 & 0.915871 & 0.899372 & 0.970569 & 0.95971 \\
\hline 2 & 0.832005 & 0.110672 & 0.047686 & 0.825399 & 0.626363 & 0.836841 \\
\hline 3 & 0.1711 & 0.138034 & 0.898131 & 0.958403 & 0.960523 & 0.301752 \\
\hline 4 & 0.699274 & 0.008757 & 0.483611 & 0.935258 & 0.840954 & 0.542465 \\
\hline 5 & 0.656374 & 0.980125 & 0.991636 & 0.551327 & 0.936239 & 0.584069 \\
\hline 6 & 0.934452 & 0.390335 & 0.348992 & 0.998471 & 0.326468 & 0.756595 \\
\hline 7 & 0.960608 & 0.201321 & 0.081413 & 0.678436 & 0.94012 & 0.927438 \\
\hline 8 & 0.994902 & 0.928847 & 0.742023 & 0.859355 & 0.48609 & 0.838213 \\
\hline 9 & 0.349416 & 0.661595 & 0.784857 & 0.972387 & 0.979741 & 0.311088 \\
\hline 10 & 0.940644 & 0.907431 & 0.431502 & 0.917884 & 0.72304 & 0.334725 \\
\hline 11 & 0.676737 & 0.91173 & 0.632703 & 0.859552 & 0.416283 & 0.982556 \\
\hline 12 & 0.173855 & 0.98662 & 0.984216 & 0.882455 & 0.895777 & 0.857537 \\
\hline 13 & 0.23628 & 0.98216 & 0.746811 & 0.948523 & 0.702253 & 0.909711 \\
\hline 14 & 0.67769 & 0.98067 & 0.560449 & 0.428774 & 0.980583 & 0.892104 \\
\hline 15 & 0.922323 & 0.285407 & 0.888201 & 0.614223 & 0.990567 & 0.981231 \\
\hline 16 & 0.13197 & 0.353394 & 0.308504 & 0.981171 & 0.40977 & 0.98075 \\
\hline 17 & 0.858765 & 0.734205 & 0.139543 & 0.901637 & 0.749911 & 0.936567 \\
\hline 18 & 0.978306 & 0.937751 & 0.095483 & 0.893269 & 0.903388 & 0.833691 \\
\hline 19 & 0.522104 & 0.754865 & 0.653823 & 0.960398 & 0.785075 & 0.450306 \\
\hline 20 & 0.59909 & 0.547994 & 0.719087 & 0.85948 & 0.513877 & 0.48225 \\
\hline 21 & 0.034842 & 0.821421 & 0.818369 & 0.90422 & 0.927915 & 0.876003 \\
\hline 22 & 0.843923 & 0.96067 & 0.461749 & 0.963881 & 0.328237 & 0.844463 \\
\hline 23 & 0.638644 & 0.415375 & 0.098925 & 0.962259 & 0.935285 & 0.447114 \\
\hline 24 & 0.960856 & 0.914799 & 0.901413 & 0.794028 & 0.985514 & 0.867843 \\
\hline 25 & 0.925466 & 0.958192 & 0.606373 & 0.940252 & 0.934111 & 0.205787 \\
\hline 26 & 0.999468 & 0.806971 & 0.9972 & 0.498365 & 0.935895 & 0.433524 \\
\hline 27 & 0.441978 & 0.670574 & 0.016995 & 0.160743 & 0.945823 & 0.51683 \\
\hline 28 & 0.790808 & 0.573207 & 0.729877 & 0.976389 & 0.581496 & 0.71045 \\
\hline 29 & 0.513427 & 0.83523 & 0.710855 & 0.864404 & 0.457556 & 0.995759 \\
\hline 30 & 0.989671 & 0.311045 & 0.905415 & 0.90395 & 0.202445 & 0.226913 \\
\hline 31 & 0.856419 & 0.433348 & 0.931232 & 0.980199 & 0.996932 & 0.212785 \\
\hline
\end{tabular}




\begin{tabular}{|c|c|c|c|c|c|c|}
\hline 32 & 0.005381 & 0.670057 & 0.264093 & 0.884975 & 0.587797 & 0.661596 \\
\hline 33 & 0.363745 & 0.007915 & 0.798633 & 0.974247 & 0.22636 & 0.972224 \\
\hline 34 & 0.096152 & 0.615228 & 0.217905 & 0.910246 & 0.378433 & 0.994777 \\
\hline 35 & 0.532371 & 0.206332 & 0.307541 & 0.889468 & 0.438639 & 0.988436 \\
\hline 36 & 0.993853 & 0.33871 & 0.17304 & 0.833497 & 0.616671 & 0.99832 \\
\hline 37 & 0.386475 & 0.73087 & 0.998458 & 0.988228 & 0.152464 & 0.922792 \\
\hline 38 & 0.581466 & 0.867274 & 0.428122 & 0.715737 & 0.560512 & 0.817602 \\
\hline 39 & 0.333348 & 0.976748 & 0.909318 & 0.323896 & 0.593916 & 0.183138 \\
\hline 40 & 0.876189 & 0.810738 & 0.056271 & 0.694105 & 0.960633 & 0.94951 \\
\hline 41 & 0.296649 & 0.88492 & 0.035896 & 0.898011 & 0.395502 & 0.150418 \\
\hline 42 & 0.744469 & 0.944458 & 0.846754 & 0.976149 & 0.938289 & 0.921875 \\
\hline 43 & 0.950693 & 0.542845 & 0.134706 & 0.827293 & 0.861363 & 0.885615 \\
\hline 44 & 0.684222 & 0.432843 & 0.703281 & 0.807887 & 0.963487 & 0.904422 \\
\hline 45 & 0.265877 & 0.638113 & 0.627033 & 0.555306 & 0.64623 & 0.23932 \\
\hline 46 & 0.383741 & 0.774615 & 0.232844 & 0.95515 & 0.989927 & 0.667583 \\
\hline 47 & 0.807276 & 0.942794 & 0.368375 & 0.985203 & 0.906295 & 0.202853 \\
\hline 48 & 0.50645 & 0.969056 & 0.746163 & 0.744025 & 0.638388 & 0.961279 \\
\hline 49 & 0.006548 & 0.989587 & 0.555021 & 0.960507 & 0.93212 & 0.966959 \\
\hline 50 & 0.158204 & 0.140217 & 0.74727 & 0.939494 & 0.821832 & 0.977984 \\
\hline
\end{tabular}

Table 7. Testing data sets for magnetic field (110kV OHTL)

\begin{tabular}{|c|c|c|c|c|}
\hline $\mathrm{x}$ & $\mathrm{y}$ & $\mathrm{He}$ & $\begin{array}{c}\mathrm{He} \\
(\mathrm{CS}-\mathrm{NN})\end{array}$ & $\begin{array}{c}\text { He } \\
\text { (proposed method) }\end{array}$ \\
\hline 0 & 5 & 0.680792 & 0.596972 & 0.907385 \\
\hline 2 & 2 & 0.110672 & 0.301365 & 0.411713 \\
\hline 5 & 2 & 0.980125 & 0.410075 & 0.607799 \\
\hline 13 & 0 & 0.23628 & 0.801731 & 0.55679 \\
\hline 14 & 2 & 0.98067 & 0.713082 & 0.706235 \\
\hline 16 & 0 & 0.13197 & 0.791938 & 0.550705 \\
\hline 17 & 5 & 0.139543 & 0.520015 & 0.677181 \\
\hline 20 & 0 & 0.59909 & 0.730082 & 0.573192 \\
\hline 22 & 0 & 0.843923 & 0.704044 & 0.834652 \\
\hline 23 & 0 & 0.638644 & 0.69793 & 0.895444 \\
\hline 27 & 2 & 0.670574 & 0.561553 & 0.582789 \\
\hline 33 & 0 & 0.363745 & 0.54131 & 0.550163 \\
\hline 40 & 2 & 0.810738 & 0.677061 & 0.949398 \\
\hline 46 & 2 & 0.774615 & 0.751552 & 0.678015 \\
\hline 49 & 0 & 0.006548 & 0.477473 & 0.030879 \\
\hline 50 & 2 & 0.140217 & 0.838409 & 0.23785 \\
\hline
\end{tabular}


Here, the magnetic field values for several new geometries are estimated and formed as a data set for training the neural network. Then the electric field of the transmission line values are evaluated for several new geometries. By applying the proposed method, the magnetic field dataset of $110 \mathrm{kV}$ transmission line is estimated and is applied for training the NN which is tabulated in Table 4. And also, the electric field is examined and tabulated in Table 5. Similarly, the electric and magnetic field values are determined by using the CS algorithm- NN controller.

Table 8. Testing data sets for electric field (110kV OHTL)

\begin{tabular}{|c|c|c|c|c|}
\hline $\mathrm{x}$ & $\mathrm{y}$ & $\mathrm{Ee}$ & $\mathrm{Ee}(\mathrm{CS}-\mathrm{NN})$ & $\begin{array}{c}\text { Ee } \\
\text { (proposed method) }\end{array}$ \\
\hline 0 & 5 & 0.896318 & 0.701468 & 0.878384 \\
\hline 2 & 2 & 0.626363 & 0.821291 & 0.786044 \\
\hline 5 & 2 & 0.936239 & 0.769309 & 0.844694 \\
\hline 13 & 0 & 0.948523 & 0.716992 & 0.755737 \\
\hline 14 & 2 & 0.980583 & 0.749322 & 0.800426 \\
\hline 16 & 0 & 0.981171 & 0.720208 & 0.760828 \\
\hline 17 & 5 & 0.936567 & 0.781834 & 0.794069 \\
\hline 20 & 0 & 0.85948 & 0.748284 & 0.87633 \\
\hline 22 & 0 & 0.963881 & 0.764822 & 0.849627 \\
\hline 23 & 0 & 0.962259 & 0.773075 & 0.88784 \\
\hline 27 & 2 & 0.945823 & 0.66013 & 0.637044 \\
\hline 33 & 0 & 0.974247 & 0.800511 & 0.780812 \\
\hline 40 & 2 & 0.960633 & 0.61742 & 0.642404 \\
\hline 46 & 2 & 0.989927 & 0.822026 & 0.838124 \\
\hline 49 & 0 & 0.960507 & 0.919525 & 0.957955 \\
\hline 50 & 2 & 0.821832 & 0.888833 & 0.87204 \\
\hline
\end{tabular}

From the above illustration, the traditional method has the relative error for the magnetic field at about 0.35 . Whereas for the proposed method the relative error for the magnetic field is about 0.05. However, from the above Figure it is clear that the relative power error of the proposed method is very much lower than that of the traditional method. Therefore, the magnetic field is highly minimized using the proposed method when compared to the existing methods. Similarly, the electric field is highly minimized using the proposed method vis-a-vis the existing methods. 


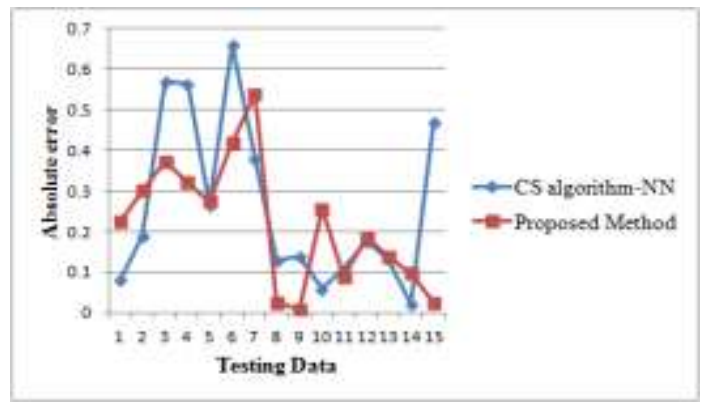

(a)

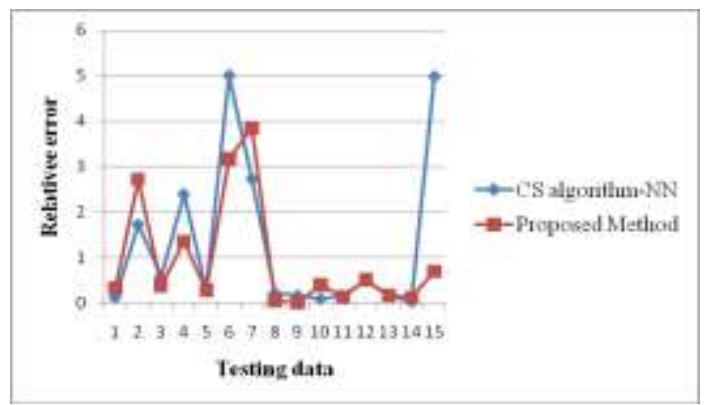

(b)

Figure 8. Comparison analysis of (a) absolute error and (b) relative error in magnetic field

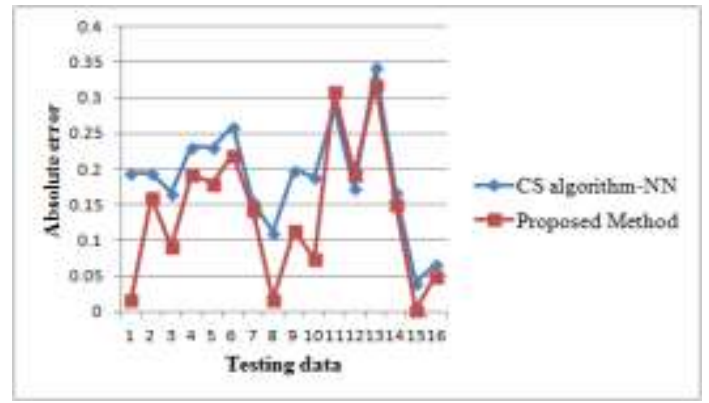

(a)

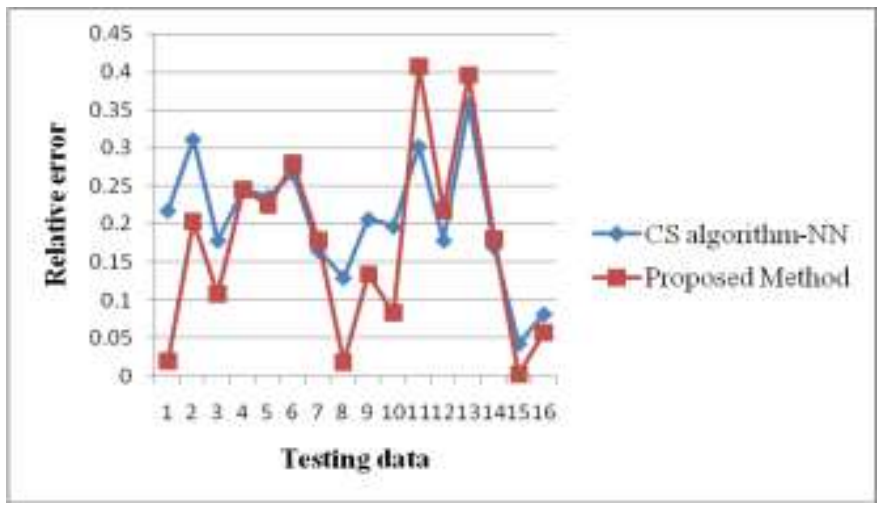

(b)

Figure 9. Comparison analysis of (a) absolute error and (b) relative error in electric field 


\section{Conclusion}

In the paper, a CS-NFC technique is proposed for estimating the electric and magnetic field of the transmission line. The proposed method is implemented in the Matlab platform. In order to obtain the minimal EMF, the arrangements of the conductor should be optimized. Initially, the $\mathrm{x}$ and $\mathrm{y}$ coordinates are given as input to the proposed method and the corresponding EMF is evaluated by using the proposed method. Then the performance of the proposed method is evaluated and analyzed with the existing methods. From the analysis, it is observed that the results of the transmission line can ensure the minimal EMF by optimizing the conductor arrangements. Therefore, by the optimal arrangement of the conductors by using proposed method, the minimal EMF is achieved when compared to the other technique. In addition, the error measurement confirms the effectiveness of the proposed method.

\section{References}

[1]. Anastasia S. Safigianni and Anastasia Kostopoulou, "Electric and magnetic field measurements in an indoor electric power substation", Journal of Materials Processing Technology, Vol.181, pp.126-130, 2007

[2]. Sol M. Michaelson, "Influence of Power Frequency Electric and Magnetic Fields on Human Health", Annals of the New York Academy of Sciences, Vol.502, pp.55-75, 1987

[3]. Anastasia S. Safigianni, and Christina G. Tsompanidou, "Measurements of electric and magnetic fields due to the operation of indoor power distribution substations", IEEE Transactions on Power Delivery, Vol.20, No.3, pp.1800-1805, July 2005

[4]. James R. Jauchem, "Exposure to extremely-low-frequency electromagnetic fields and radiofrequency radiation: cardiovascular effects in humans", International Archives of Occupational and Environmental Health, Vol.70, No.1, pp.9-21, 1997

[5]. A. Babouri and W. Tourab, M. Nemamcha, "A study of the electromagnetic environment in the vicinity of high-voltage lines" In the proceedings of Power Engineering, Energy and Electrical Drives, pp.1181-1184, 2013

[6]. Wong, P.S., Rind, T.M., Harvey, S.M., and Scheer, R.R., "Power frequency electric and magnetic fields from a $230 \mathrm{kV}$ gas-insulated substation", IEEE Transactions on Power Delivery, Vol.9, No.3, pp.1494-1501, 1994

[7]. Zipse, Donald W., "Health effects of extremely low-frequency (50 and $60 \mathrm{~Hz}$ ) electric and magnetic fields", IEEE Transactions on Industry Applications, Vol.29, No.2, pp.447458,1993

[8]. S. Nikolovski, Z. Klaic, Z. Kraus, and M. Stojkov, "Computation and measurement of electromagnetic fields in high voltage transformer substations", MIPRO 2010, 2010

[9]. Munteanu, V. Topa, A. Racasan, G. Visan, and I. T. Pop, "Computation Methods and Experimental Measurements of the Electric and Magnetic Field Distribution inside High Voltage Substations", International Conference on Electromagnetics in Advanced Applications, 2009

[10]. W. K. Daily, and F. Dawalibi, "Measurements and Computations of Electromagnetic Fields in Electric Power Substations", IEEE Transactions on Power Delivery, Vol.9, No.1, pp.324-333, 1994

[11]. S. Nikolovski, Z. Klaic, and B. Stefic, "Measurements and Computation of Electromagnetic Field in Transformer Station 400/110 kV Ernestinovo", IEEE Bucharest PowerTech, 2009

[12]. Lucio Ippolito, and Pierluigi Siano, "Using multi-objective optimal power flow for reducing magnetic fields from power lines", Electric Power Systems Research, Vol.68, No.2, pp.93-101, 2003

[13]. Jasna J. Radulovic, and Vesna M. Rankovic, "Application of RBF Neural Network and ANFIS for Electromagnetic Field Prediction around the Power Lines", 9th International Conference on Telecommunication in Modern Satellite, Cable, and Broadcasting Services, 2009 
[14]. R.K Aggarwal, A.T Johns, J.A.S.B Jayasinghe, and W Su, "An overview of the condition monitoring of overhead lines", Electric Power Systems Research, Vol.53, No.1, pp.15-22, 2000

[15]. S.J. Gusavac, M.D. Nimrihter, and Lj.R. Geric, "Estimation of overhead line condition", Electric Power Systems Research, Vol.78, pp.566-583, 2008

[16]. Lee, M.A., and Takagi, H., "Integrating design stage of fuzzy systems using genetic algorithms", IEEE International Conference on Fuzzy Systems, Vol.1, pp.612-617, 1993

[17]. Yaochu Jin, "Fuzzy modeling of high-dimensional systems: complexity reduction and interpretability improvement", IEEE Transactions on Fuzzy Systems, Vol.8, No.2, pp.212221,2000

[18]. L Ekonomou, V T Kontargyri, St Kourtesi, T I Maris, and I A Stathopulos, "Artificial neural networks in high voltage transmission line problems", Measurement Science and Technology, Vol.18 No.7, 2007

[19]. Isik Yilmaz, and Oguz Kaynar, "Multiple regression, ANN (RBF, MLP) and ANFIS models for prediction of swell potential of clayey soils", Expert Systems with Applications, Vol.38, No.5, pp.5958-5966, 2011

[20]. Wang Jeen-Shing, and Lee C.S.G, "Self-adaptive neuro-fuzzy inference systems for classification applications", IEEE Transactions on Fuzzy Systems, Vol.10, No.6, pp.790802,2002

[21]. Jasna Radulovic, and Vesna Rankovic, "Feedforward neural network and adaptive network-based fuzzy inference system in study of power lines", Expert Systems with Applications, Vol.37, pp.165-170, 2010

[22]. Zemljaric, B., "Calculation of the Connected Magnetic and Electric Fields Around an Overhead-Line Tower for an Estimation of Their Influence on Maintenance Personnel", IEEE Transactions on Power Delivery, Vol.26, No.1, pp.467-474, 2011

[23]. Jasna Radulovic, and Vesna Rankovic, "Prediction of magnetic field near power lines by normalized radial basis function network", Advances in Engineering Software, Vol. 42, pp. 934-938, 2011

[24]. Xu Sun, Qi Huang, Yunhe Hou, Lijun Jiang, and Pong, P.W.T., "Noncontact Operation State Monitoring Technology Based on Magnetic Field Sensing for Overhead High Voltage Transmission Lines", IEEE Transactions on Power Delivery, Vol.28, No.4, pp.2145-2153, 2013

[25]. F. Munoz, J.A. Aguado, F. Martin, J.J. Lopez, A. Rodriguez, J.B. Garcia, A.R. Treitero, and R. Molina, "An intelligent computing technique to estimate the magnetic field generated by overhead transmission lines using a hybrid GA-Sx algorithm", Electrical Power and Energy Systems, Vol.53, pp.43-53, 2013

[26]. M. S. H. Al Salameh and M. A. S. Hassouna, "Arranging Overhead Power Transmission Line Conductors Using Swarm Intelligence Technique to minimize Electromagnetic Fields", In proceedings of Electro Magnetics Research, Vol. 26, pp. 213-236, 2010

[27]. M. RamaMohana Rao and A. V. Naresh Babu, "Optimal Power Flow Using Cuckoo Optimization Algorithm", International Journal of Advanced Research in Electrical, Electronics and Instrumentation Engineering, Vol. 2, No. 9, September 2013

[28]. Farzan Rashidi and Behzad Moshiri, "Improvement of Low Frequency Oscillation Damping in Power Systems via an Adaptive Critic Based Neuro Fuzzy Controller", In proceedings of Knowledge-Based Intelligent Information and Engineering Systems Lecture Notes in Computer Science, Vol. 3215, pp. 559-565, 2004 